\title{
Gamma-Ray Bursts via Pair Plasma Fireballs from Heated Neutron Stars
}

\author{
Jay D. Salmonson*, James R. Wilson*† and Grant J. Mathews ${ }^{\dagger}$ \\ * Lawrence Livermore National Laboratory, Livermore, CA 94550 \\ $\dagger$ University of Notre Dame, Notre Dame, IN 46556
}

\begin{abstract}
In this paper we model the emission from a relativistically expanding $e^{+} e^{-}$ pair plasma fireball originating near the surface of a heated neutron star. This pair fireball is deposited via the annihilation of neutrino pairs emanating from the surface of the hot neutron star. The heating of neutron stars may occur in close neutron star binary systems near their last stable orbit. We model the relativistic expansion and subsequent emission of the plasma and find $\sim 10^{51}-10^{52}$ ergs in $\gamma$-rays are produced with spectral and temporal properties consistent with observed gamma-ray bursts.
\end{abstract}

\section{INTRODUCTION}

It has been speculated for some time that inspiraling neutron stars could provide a power source for cosmological gamma-ray bursts [5,6]. However, previous Newtonian and post-Newtonian studies [1] of the final merger of two neutron stars have found that the neutrino emission time scales are so short that it would be difficult to drive a gamma-ray burst from this source. It is clear that a mechanism is required for extending the duration of energetic neutrino emission. A number of possibilities could be envisioned, for example, neutrino emission powered by accretion shocks, MHD or tidal interactions between the neutron stars, etc. The present study, however, has been primarily motivated by numerical studies of the strong field relativistic hydrodynamics of close neutron star binaries (NSBs) in three spatial dimensions. These studies $[11,12,4,2]$ suggest that neutron stars in a close binary can experience relativistic compression and heating over a period of seconds. During the compression phase released gravitational binding energy can be converted into internal energy. Subsequently, up to $10^{53}$ ergs in thermally produced neutrinos can be emitted before the stars collapse [4]. Here we briefly summarize the physical basis of this model and numerically explore its consequences for the development of an $e^{+} e^{-}$plasma and associated GRB.

In [4] properties of equal-mass neutron-star binaries were computed as a function of mass and EOS (Equation of State). From these studies it was deduced that compression, heating and collapse could occur a few seconds before binary merger. 
Our calculation of the rates of released binding energy and neutron star cooling suggests that interior temperatures as hot as $70 \mathrm{MeV}$ are achieved. This leads to a high neutrino luminosity which peaks at $L_{\nu} \sim 10^{53} \mathrm{ergs} \mathrm{sec}^{-1}$. This much neutrino luminosity would partially convert to an $e^{+} e^{-}$pair plasma above the stars as is also observed above the nascent neutron star in supernova simulations [13].

\section{NEUTRINO ANNIHILATION AND PAIR CREATION}

Having outlined a mechanism by which neutrino luminosities of $10^{52}$ to $10^{53}$ ergs/sec may arise from binary neutron stars approaching their final orbits, we must calculate the efficiency of conversion of neutrino pairs into an electron pair plasma via $\nu \bar{\nu} \rightarrow e^{+} e^{-}$. Here we argue that the efficiency for converting these neutrinos into pair plasma is probably quite high. Neutrinos emerging from the stars will deposit energy outside the stars predominantly by $\nu \bar{\nu}$ annihilation to form electron pairs. A secondary mechanism for energy deposition is the scattering of neutrinos from the $e^{+} e^{-}$pairs. Strong gravitational fields near the stars will bend the neutrino trajectories. This greatly enhances the annihilation and scattering rates [8]. For our employed neutron-star equations of state the radius to mass ratio is typically between $R / M \sim 3$ and 4 just before stellar collapse (in units $G=c=1$ ). In [8] it is shown that $\nu \bar{\nu}$ annihilation rates will be enhanced by a factor $\mathcal{F}(R / M) \sim 8$ to 28 due to relativistic effects. From Eq. 24 of [8] we obtain,

$$
\frac{\dot{Q}}{L_{\nu}} \approx 0.03 \mathcal{F}(R / M) L_{53}^{5 / 4} \text {. }
$$

Thus, the efficiency of annihilation ranges from $\approx 0.1$ to $0.84 \times L_{53}^{5 / 4}$. For the upper range of luminosity the efficiency is quite large. Also, using the supernova code of Wilson and Mayle [13] we calculate the entropy per baryon of the plasma to be as high as $10^{6}$, thus the resulting pair plasma will have low baryon loading.

\section{PAIR PLASMA EXPANSION AND SHOCK WITH ISM}

Having determined the initial conditions of the hot $e^{+} e^{-}$pair plasma near the surface of a neutron star, we wish to follow its evolution and characterize the observable gamma-ray emission. To study this we have developed a spherically symmetric, general relativistic hydrodynamic computer code to track the flow of baryons, $e^{+} e^{-}$pairs, and photons. For the present discussion we consider the plasma deposited at the surface of a $1.45 M_{\odot}$ neutron star with a radius of $10 \mathrm{~km}$. Discussion of this code can be found in $[9,7]$. In those papers the emission from an expanding fireball was studied. In Figure 1 it is shown that the resulting emission spectrum and $\gamma$-ray emission efficiency $E_{\gamma} / E_{\text {tot }}$ strongly depends upon the entropy per baryon of the plasma deposited near the surface of the neutron stars; entropies of $\lesssim 10^{6}$ resulted in weak emission with most of the original energy manifesting 


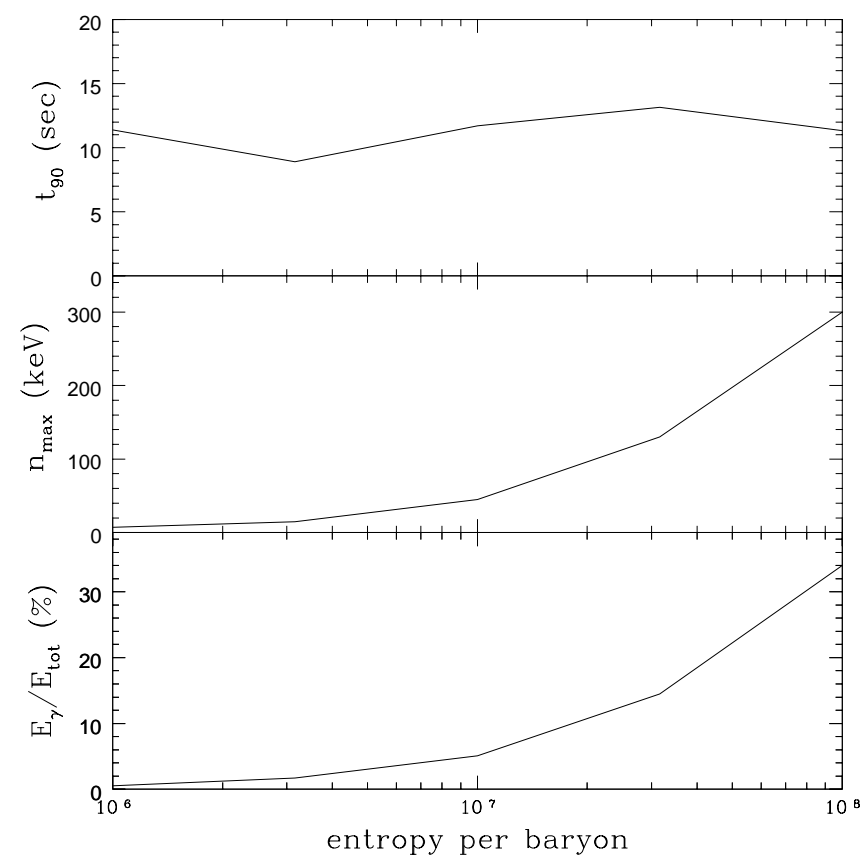

FIGURE 1. The duration, energy at the number spectrum peak, and gamma-ray efficiency are plotted for the emission from an expanding pair plasma with total deposited energy $E_{t o t}=10^{52}$ ergs over a range of entropies per baryon $10^{6}$ to $10^{8}$.

itself as kinetic energy of the baryons. Thus, for the low entropy per baryon fireballs $\left(s \sim 10^{5}-10^{6}\right)$ produced by NSBs it is necessary to examine the emission due to the interaction of the relativistically expanding baryon wind with the interstellar medium (ISM). We find that these baryon winds typically have a Lorentz factor $\gamma \approx 300$ and have a total energy $\approx 10^{52}$ ergs.

After becoming optically thin and decoupling with the photons, the matter component of the fireball continues to expand and interact with the ISM via collisionless shocks. As the ISM is swept up, the matter decelerates. We model this process as an inelastic collision between the expanding fireball and the ISM as in, for example, [6]. We assume that the absorbed internal energy is immediately radiated away. From this we construct a simple picture of the emission due to the matter component of the fireball "snowplowing" into the ISM of baryon number density $n$.

We have constructed an analytic formula for the luminosity in time [7] of the fireball plowing into the ISM. We show a plot of this function in Figure 2 for a range of ISM densities. Defining $t_{\max }$ as the time of maximum luminosity

$$
L(t) \propto \begin{cases}t^{2} & \text { free expansion phase }\left(t<t_{\max }\right) \\ t^{-10 / 7} & \text { deceleration phase }\left(t>t_{\max }\right) .\end{cases}
$$

This luminosity curve has the so called "FRED" (Fast Rise, Exponential Decay) profile which is characteristic of real bursts. 


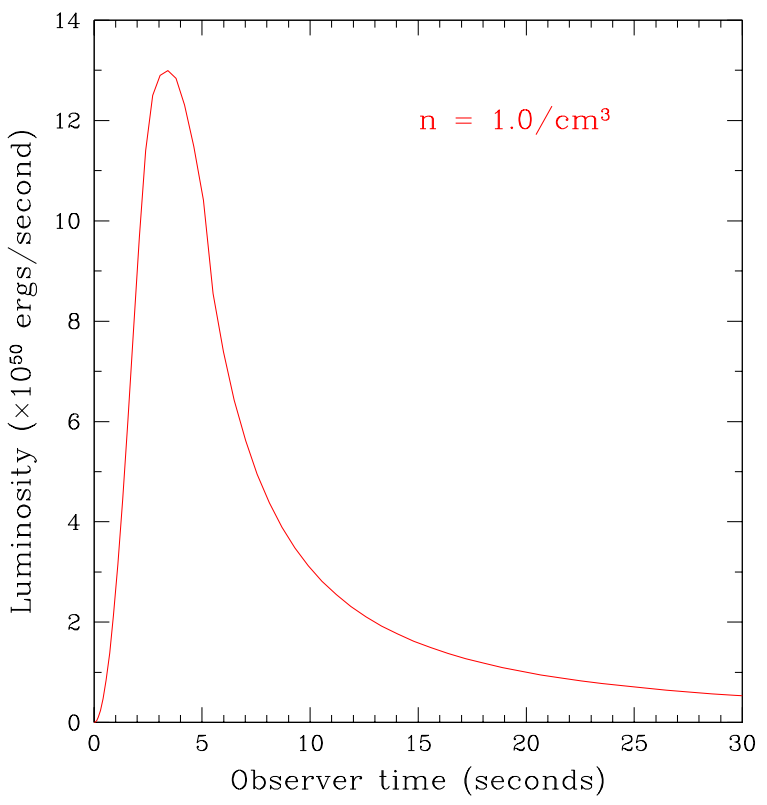

FIGURE 2. The light curve for a $10^{52}$ erg fireball expanding at $\gamma=300$ into the interstellar medium with number density of one baryon per $\mathrm{cm}^{3}$.

\section{Synchrotron Shock Spectrum}

Using the theory of synchrotron shocks (e.g. [10]) we can construct a spectrum as shown in Figure 3. To model the synchrotron spectrum there are three free parameters: $\epsilon_{B}, \epsilon_{e}$ are the fractions of baryonic kinetic energy that is deposited into the magnetic field and the electrons respectively, and $n$, the number density of baryons in the ISM. In these calculations we assume $\epsilon_{B}=\epsilon_{e}=1 / 4$. As shown in Figure 3 , a reasonable ISM density of 1 baryon $/ \mathrm{cm}^{3}$ gives a peak in the $\nu L_{\nu}$ spectrum at $\sim 100 \mathrm{keV}$ in agreement with observations. Calculations of the efficiency show that $75 \%$ of the energy is emitted at photon energies of $10 \mathrm{keV}$ and above [7].

\section{CONCLUSIONS}

In this proceedings we have argued that heated neutron stars (perhaps by stellar compression of close neutron-star binaries) are viable candidates for the production of large, high entropy per baryon, $e^{+} e^{-}$pair plasma fireballs, and thus, for the creation of gamma-ray bursts. We find that fireballs of total energies $E \sim 10^{51}$ to $3 \times 10^{52}$ ergs and entropies per baryon $s>10^{5}$ are possible. Also, this model gives a power-law spectrum that peaks at hundreds of $\mathrm{keV}$ and has an overall efficiency of $10-20 \%$.

Work performed under the auspices of the U.S. Department of Energy by the Lawrence Livermore National Laboratory under contract W-7405-ENG-48. J.R.W. 


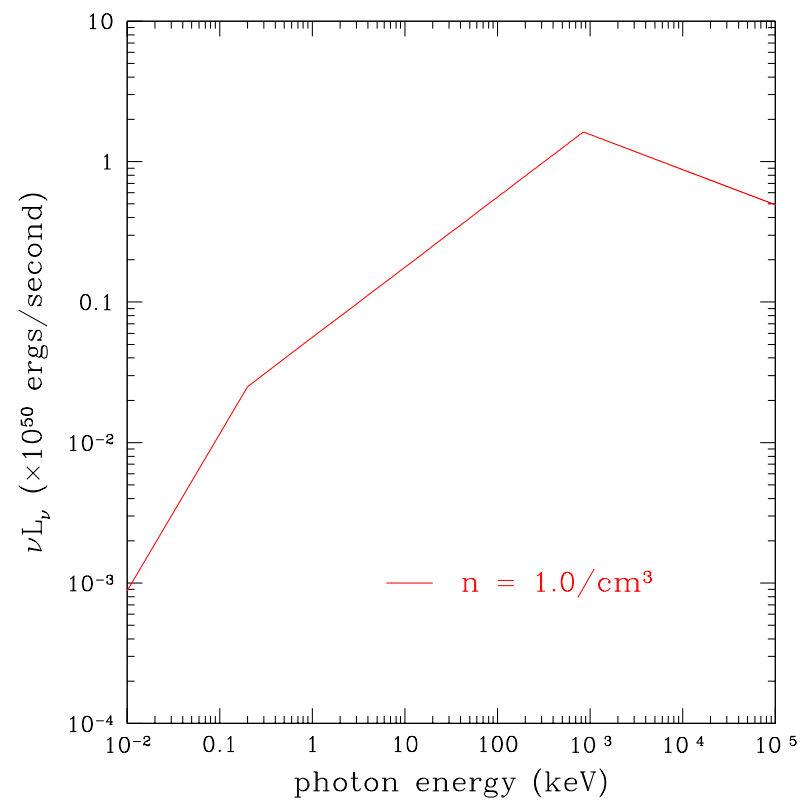

FIGURE 3. The peak synchrotron spectrum for a $10^{52}$ erg fireball expanding at $\gamma=300$ into interstellar medium number density of one baryon per $\mathrm{cm}^{3}$.

was partly supported by NSF grant PHY-9401636. Work at University of Notre Dame supported in part by DOE grant DE-FG02-95ER40934, NSF grant PHY-9722086, and by NASA CGRO grant NAG5-3818.

\section{REFERENCES}

1. Janka, H. T. and Ruffert, M., A $\& A$ 307, L33 (1996).

2. Mathews, G. J., Marronetti, P. and Wilson, J. R., Phys. Rev. D 58, 043003 (1998).

3. Mathews, G. J. and Wilson, J. R., in press Phys. Rev. D, gr-qc/9911047 (2000).

4. Mathews, G. J. and Wilson, J. R., ApJ 482, 929 (1997).

5. Mészáros, P. and Rees, M. J., ApJ 397, 570 (1992).

6. Piran, T., astro-ph/9907392 (1999).

7. Salmonson, J. D. and Wilson, J. R. and Mathews, G. J., submitted ApJ (2000).

8. Salmonson, J. D. and Wilson, J. R., ApJ 517, 859 (1999).

9. Salmonson, J. D. and Wilson, J. R. and Mathews, G. J., in Gamma-Ray Bursts: 4th Huntsville Symposium, AIP Conf. Proc. 428, eds. C. A. Meegan, R. D. Preece and T. M. Koshut, 1997, p. 788.

10. Sari R., Piran T., Narayan R., ApJL 497, L17 (1998).

11. Wilson, J. R. and Mathews, G. J., Phys. Rev. Lett. 75, 4161 (1995).

12. Wilson, J. R. and Mathews, G. J. and Marronetti, P., Phys. Rev. D 54, 1317 (1996).

13. Wilson, J. R. and Mayle, R. W., Phys. Rep. 227, 97 (1993). 